\title{
Dynamic models for multiplication and division offered by GeoGebra
}

\author{
Lindita Kllogjeri ${ }^{1, ~ *, ~ P e l l u m b ~ K l l o g j e r i ~}{ }^{1,2}$ \\ ${ }^{1}$ The General High School, 9-Year Cycle, Labinot-Fushe, Elbasan, Albania \\ ${ }^{2}$ University of Elbasan, Department of Mathematics and Informatics, Elbasan, Albania
}

\section{Email address:}

peristerikllogjeri@gmail.com (L. Kllogjeri),pkallogjeri@gmail.com (P. Kllogjeri)

\section{To cite this article:}

Lindita Kllogjeri, Pellumb Kllogjeri. Dynamic Models for Multiplication and Division Offered by GeoGebra. American Journal of Software Engineering and Applications. Special Issue: Growing GeoGebra Environment and Use. Vol. 4, No. 2-1, 2015, pp. 1-6. doi: 10.11648/j.ajsea.s.2015040201.11

\begin{abstract}
One of the most important features of GeoGebra is the coordination of the geometric and algebraic representations, easily observed in GeoGebra window. Using GeoGebra software the teacher can geometrically and fruitfully teach the concepts and algorithms of arithmetic operations in the elementary school. Our paper focuses on two important operations: multiplication and division in the set of natural numbers. Using GeoGebra features we visually demonstrate the concepts of these two operations and help the students to develop the process of mastering multiplication and division facts. Our paper aims to achieve three objectives: Firstly, teach multiplication and division using an area model with base and height of 10 squares. The table designed for this special purpose can be considered as a platform where the arrangements of objects, pictures or numbers in columns and rows is done. Secondly, teach division by using the concept of sharing or partitioning. We have designed a particular dynamic model allowing the teacher to convey the meaning of division so that the students can have a better understanding of the division process. Thirdly, by creating dynamic models for teachers and students we want to: 1. Increase teacher pedagogical content knowledge and improve the instructional practice; 2. Promote student learning by improving teaching practices and providing capacity-building solutions; 3. Encourage the teachers engage themselves in research activity and innovative educational practices and teaching strategies
\end{abstract}

Keywords: Multiplication Model, Division Model, Technology

\section{Introduction}

We are witnesses of rapid technological developments and changings in the business world, in industry, in the organization and management of learning institutions etc. Information and Communication Technology (ICT) is continually adding values in the process of teaching and learning as well. Today are available many ICT resources, almost in every country and region, such as computer laboratories, internet access, computer softwares and so on. GeoGebra software is a very good and attractive one, useful for any level of the education system and free. Technology requires that the new skills and knowledge be frequently mastered and adopted. The teachers have to be focused on gaining the ICT competencies, on understanding how and when to use ICT tools. Understanding how and when to use ICT tools is a basic stage for all the educators, managers and specialists.
This stage implies the ability to recognize situations where ICT will be helpful, choosing the most appropriate tools for a particular task, and using these tools in combination to solve real problems. (INFORMATION AND COMMUNICATION TECHNOLOGY IN EDUCATION, pp.17)

Particularly, this stage is linked with the infusing and transforming approaches in ICT development. The teachers use ICT to manage not only the learning of their students but also their own learning.

In the infusing approach to ICT development, ICT infuses all aspects of teachers' professional lives in such a way as to improve student learning and the management of learning processes. The approach supports active and creative teachers who are able to stimulate and manage the learning of students, integrating a range of preferred learning styles and uses of ICT in achieving their goals.

In the transforming approach to ICT development, teachers 
and other school staff regard ICT as so natural and part of the everyday life of schools that they begin to look at the process of teaching and learning in new ways. The emphasis changes from teacher-centred to learning centred. (INFORMATION AND COMMUNICATION TECHNOLOGY IN EDUCATION, pp.20)

GeoGebra is a dynamic software package allowing teachers and students to visualize geometric transformations and explore geometric properties. It incorporates algebra, calculus and geometry. GeoGebra can be used by teachers and students: teachers providing step-by-step instructions for the students and the students by independently using GeoGebra tools and commands to construct and explore different objects and properties and generate knowledge. Since the time GeoGebra software has been introduced and ongoing there is a huge amount of materials, tutorials and experiences and a great number of GeoGebra trainers, specialists and educators with web-based monitoring. GeoGebra provides a seamless link between the various mathematical sciences and, between mathematics and other sciences. It promotes the study of mathematics, technology, physics, biology, engineering etc. It encourages exploration, creativity and learning, by doing, in any science and in any level of the school.

One of the most important features of GeoGebra is the coordination of the geometric and algebraic representations, easily observed in GeoGebra window. Using GeoGebra software the teacher can geometrically and fruitfully teach the concepts and algorithms of arithmetic operations in the elementary school. Our paper focuses on two important operations: multiplication and division in the set of natural numbers. Multiplying and dividing are two very important math skills that all students should have. Using GeoGebra features we demonstrate and visualize the concepts of these two operations and help the students to develop the process of mastering multiplication and division facts. The students learn to use patterns and property theories as strategies for recalling the facts related to multiplication and division. They develop, by personal manipulation and exploration of patterns in multiplication and division, computational fluency. Our paper aims to achieve three objectives:

Firstly, teach multiplication and division using an area model with base and height of 10 squares. The table designed for this special purpose can be considered as a platform where the arrangements of objects, pictures or numbers in columns and rows is done.

Secondly, teach division by using the concept of sharing or partitioning. We have designed a particular dynamic model that allows the teacher to convey the meaning of division so that the students can have a better understanding of the division process.

Thirdly, by creating innovative and professional dynamic models for teachers and students we want to:

- Increase teachers pedagogical content knowledge and improve their instructional practice

- Allow the schools to use technology effectively to improve and enhance teaching and learning

- Promote student learning by improving teaching practices and providing capacity-building solutions

- Encourage the teachers engage themselves in research activity and innovative educational practices and teaching strategies

\section{The Dynamic Multiplication Model and the Benefits}

\subsection{The Dynamic Multiplication Model}

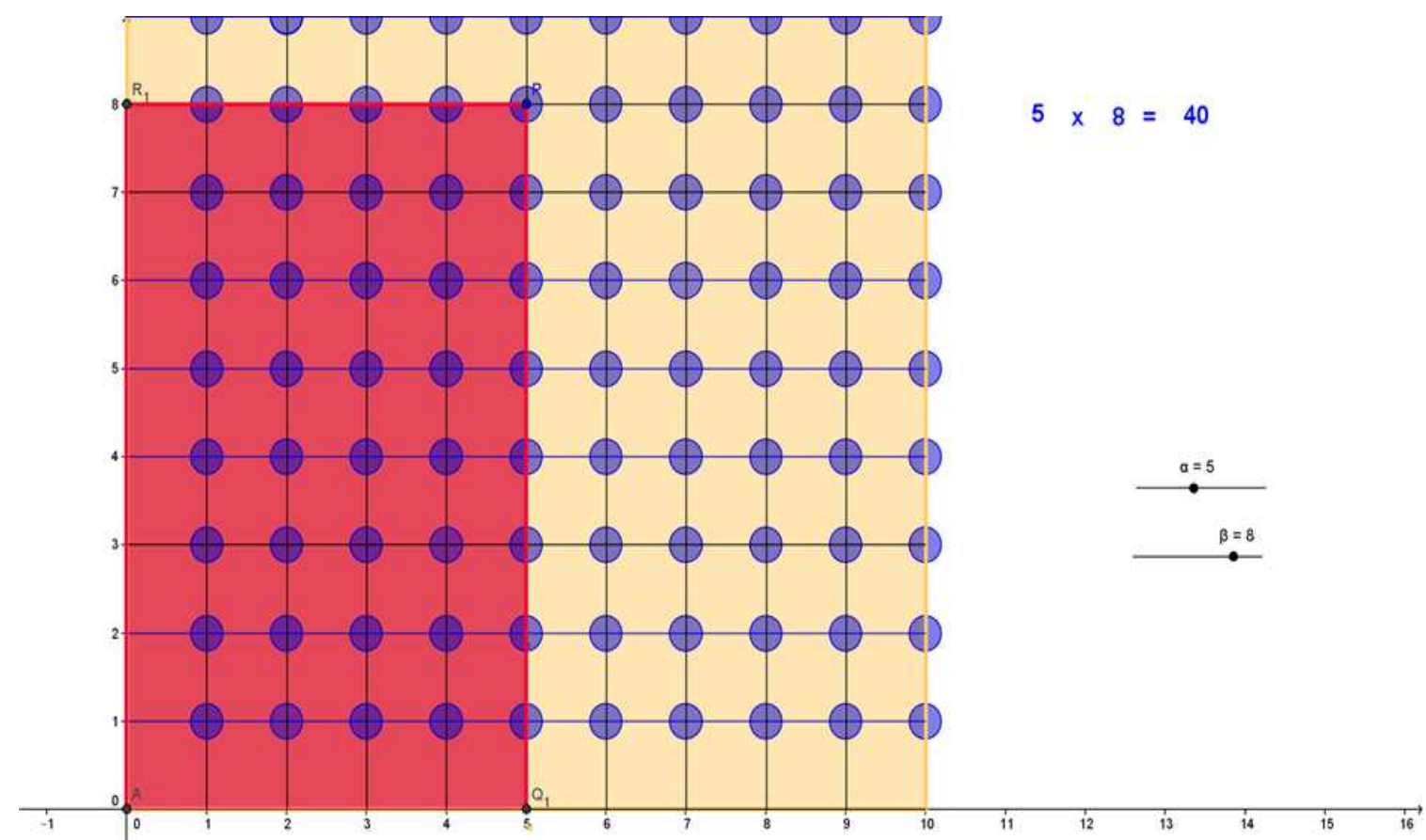

Fig. 1. Multiplication Table 
In the case of multiplication: students are given length and width of a rectangular to find its area which is the product (length $\mathrm{x}$ (times) width or factor $\mathrm{x}$ (times) factor). The figure above shows the case of calculating the area of the rectangular (red color) of base: $\alpha=5$ units and hight: $\beta=8$ units. The respective area is: $a=\alpha \beta=5 \times 8=40$ square units. Instead of calculating the area can be assigned the task of finding the number of the circles arranged in a rectangular array of 8 rows and 5 columns, or arranged in a rectangular array of 5 rows and 8 columns (this relates to another figure).

The table designed for this special purpose can be considered as a platform where the arrangements of objects, pictures or numbers in columns and rows is done (Look at Fig. 1). This is an array method of explaining the multiplication. On the other side, this array is used for the concept of division related to multiplication as its inverse operation. In the case of division: students are given area and the length in order to find the width (that is: given dividend and the divisor let be found the quotient). Also, the students learn how to use arrays to show the relationship between multiplication and division.

\subsection{Procedure of Constructing the Multiplication Table}

1. Construct a square $10 \times 10$ with left lower vertex at the origin of the coordinative system and fill it with a colour (for example, gold).

2. Construct its respective coordinate grid $10 \times 10$ (the crossed segments coincide with the coordinative lines).

3. Insert two sliders:

- $\alpha$ with $\min =1, \max =10$ and increment 1

- $\beta$ with $\min =1, \max =10$ and increment 1

4. Insert into the Input field the point $\mathrm{P}=(\alpha, \beta)$

5. Construct the rectangle with diagonal $\mathrm{OP}\left(\mathrm{OQ}_{1} \mathrm{PR}_{1}\right)$ and fill it with red colour.

6. Insert into the Input field, one at a time, the variables $\alpha$ and $\beta$ and the equation $v=\alpha \cdot \beta$ ( $\alpha$ and $\beta$ represent the factors of the product: $v=\alpha \cdot \beta)$.

7. Insert the following texts (each one separately):

- $\alpha$ (representing factor)

- $\beta$ (representing factor)

- X (representing the multiplication operation)

- = (representing the equality sign)

- $v$ (representing the product)

8. For each text choose properties to select the colour (blue), the size of the text etc.

9. Arrange the texts on the right side of the square grid to be on a row, and form the equation $\beta=v$.

The value of the product corresponds to the respective values of $\alpha$ and $\beta$ on their respective sliders.

10. Change the values of $\alpha$ and $\beta$ on their respective sliders and observe their product at the equation (for example: 5 X $8=40$ ).

Interpretation and observation: interpret the result of the product referring to the number of the unit squares consisting the rectangle $O Q_{1} P_{1}$, or refer to its area. Manipulate the sliders and observe the product at the equation and the respective rectangle with diagonal OP. You can change the position of point $\mathrm{P}$ and observe the change of $\alpha$ and $\beta$ on their respective sliders. The dependence between the product $d$ the values of $\alpha$ and $\beta$, in our case, is bidirectional.

\subsection{Another Interpretation of the Multiplication Operation}

Additional procedure:

1. Using the virtual tool for circle, construct circles with radius 0.2 and with centre at each intersection point of the grid (look at figure above). There are 100 such circles.

2. Using object properties of each circle, colour them blue. Interpretation and observation: interpret the result of the product of the whole numbers $m$ and $n$ as the total number of the circles (objects) arranged in a rectangular array, consisted of $\mathrm{m}$ horizontal rows of $\mathrm{n}$ circles (objects) each and $\mathrm{n}$ vertical columns of $\mathrm{m}$ circles (objects) each, e.g. interpret $5 \times 8$ as the total number of the circles in 8 horizontal rows of 5 circles each.

Differently, interpret the result of the product of the whole numbers as the total number of the circles (objects) in 5 vertical rows of 8 circles (objects) each.

Note: The multiplication model can be used to compute the product of decimal numbers of our base-ten number system, as well. For numbers having one digit to the right of the decimal point choose at the sliders increment 0.1 . For numbers having two digits to the right of the decimal point choose at the sliders increment 0.01 and so on. In this case the product result relates to the approximate area of the rectangle $\mathrm{OQ}_{1} \mathrm{PR}_{1}$.

The multiplication model can be used as a calculator. If we want to approximately calculate $3.4 \times 5.6$ then choose the values $\alpha=3.4$ and $\beta=5.6$ at the sliders and read the approximate product at the equation in geometry window or at poly 2 , or at $v$ in algebra window. Another way: using Move tool displace the point $\mathrm{P}$ in such a way that its coordinates be 3.4 and 5.6. Check the result at the equation.

Visit http://tube.geogebra.org/material/show/id/134094 to play with the model.

\subsection{The Benefits of Using the Multiplication Model}

The teachers:

- Provide professional development

- Improve teaching practices

- Use innovative educational practices and technologies

- Provide capacity-building solutions through the use of GeoGebra software

- Integrate mathematics pedagogy and technology

- Engage in research activity in the areas of educational content, instruction and technology

- Improve and enhance teaching and student learning

- Help in developing policies and programs

- Extension activities: construct multiplication table through $12 \times 12,15 \times 15$ etc.

- Design multiplication games

The students:

- Get clear meaning about the result of the multiplication 
- Develop computational fluency

- Master multiplication facts: zero property, one property, commutative property, multiples of 2 , multiples of 5 etc.

- Model their thinking and develop mind patterns for multiplication operation

- Develop fluency in multiplying whole numbers

- Use and develop strategies to estimate the results of multiplication and judge the reasonableness of such results.

- Understand and apply the properties of multiplication operation

\section{The Division Model}

\subsection{Partition Model}

The division can be thought of in two ways: partitioning and measurement. We will build a model related to the first concept, partitioning. This relates to the case of finding the number of objects in each group when the total number of the objects is shared in equal quantities. The performed operation is called fair sharing or partitioning. The other case relates to finding the number of groups and the division is called measuring or repeated subtraction. For instance, we keep subtracting 3 objects from 15 objects until we reach zero. Each 3 subtracted is a group.

Let us be concentrated on partitioning. Using the following model we can convey the meaning of the first kind of division in order the students have a better understanding of the division process. The model presented is valid for the interpretation of the whole-number quotient of a whole number. For every case of a division "whole-number quotient of a whole number" must be designed a model which helps to interpret the case. We have chosen the case of partitioning the set of 12 objects into:

(a) 2 groups of equal shares in each one, symbolically denoted as $12 \div 2$

(b) 3 groups of equal shares in each one, symbolically denoted as $12 \div 3$

(c) 4 groups of equal shares in each one, symbolically denoted as $12 \div 4$

The 12 objects are represented by 12 blue circles (look at Fig.2). The respective model for the division interpretation is constructed using GeoGebra software with its effective virtual tools and the excellent periodic properties of the trigonometric functions. The model of this case and the respective procedure serve as a demonstrative model for the teachers to use in their classroom, equipped with computers, or in computer laboratories. By watching this case the students are able to figure out and build in their minds similar models for other cases of division "whole-number quotient of a whole number". Manipulatives and visual aids are very important when teaching multiplication and division.

On the other side, the teachers can use the presented procedure to construct models for other cases of the division. Furthermore, they can design and develop other procedures using GeoGebra software and the properties of the trigonometric functions or recursive sequences.

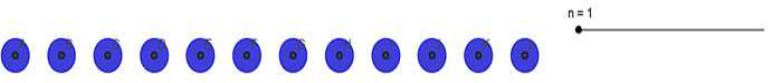

$12: 1=12$

Fig. 2. The set of 12 objects grouped together

\subsection{The procedure for the Division Model}

Case: $12 \div \mathrm{n} ; \mathrm{n}=2,3,4$

1. Open GeoGebra window

2. Construct a slider with parameter $\mathrm{n}$; interval $[1,4]$, increment 1

3. Successively enter in the Input field the following points:

$$
\begin{gathered}
A=\left\{1,8-2 \cdot\left[1-\left(\sin \left(\frac{n \pi}{2}\right)\right)^{2 n}-\left(\cos \left(\frac{n \pi}{2}\right)\right)^{2 n}\right]\right\} \\
B=\left\{2,8-2 \cdot\left[1-\left(\sin \left(\frac{n \pi}{2}\right)\right)^{2 n}-\left(\cos \left(\frac{n \pi}{2}\right)\right)^{2 n}\right]\right\} \\
C=\left\{3,8-2 \cdot\left[1-\left(\sin \left(\frac{n \pi}{2}\right)\right)^{2 n}-\left(\cos \left(\frac{n \pi}{2}\right)\right)^{2 n}\right]\right\} \\
D=\left\{4,8-2 \cdot\left[1-\left(\sin \left(\frac{n \pi}{2}\right)\right)^{2 n}+\left(\cos \left(\frac{n \pi}{2}\right)\right)^{2 n+1}\right]\right\} \\
E=\left\{5,8-2 \cdot\left[1-\left(\sin \left(\frac{n \pi}{2}\right)\right)^{2 n+1}+\left(\cos \left(\frac{n \pi}{2}\right)\right)^{2 n+1}\right]\right\} \\
F=\left\{6,8-2 \cdot\left[1-\left(\sin \left(\frac{n \pi}{2}\right)\right)^{2 n+1}+\left(\cos \left(\frac{n \pi}{2}\right)\right)^{2 n+1}\right]\right\} \\
G=\left\{7,8-2 \cdot\left[1-\left(\sin \left(\frac{n \pi}{2}\right)\right)^{2 n+1}-\left(\cos \left(\frac{n \pi}{2}\right)\right)^{2 n+1}\right]\right\} \\
H=\left\{8,8-2 \cdot\left[1-\left(\sin \left(\frac{n \pi}{2}\right)\right)^{2 n+1}-\left(\cos \left(\frac{n \pi}{2}\right)\right)^{2 n+1}\right]\right\} \\
I=\left\{9,8-2 \cdot\left[1-\left(\sin \left(\frac{n \pi}{2}\right)\right)^{2 n}-\left(\cos \left(\frac{n \pi}{2}\right)\right)^{2 n+1}\right]\right\} \\
J=\left\{10,8-2 \cdot\left[1-\left(\sin \left(\frac{n \pi}{2}\right)\right)^{2 n}+\left(\cos \left(\frac{n \pi}{2}\right)\right)^{2 n}\right]\right\}
\end{gathered}
$$


$K=\left\{11,8-2 \cdot\left[1-\left(\sin \left(\frac{n \pi}{2}\right)\right)^{2 n}+\left(\cos \left(\frac{n \pi}{2}\right)\right)^{2 n}\right]\right\}$

$L=\left\{12,8-2 \cdot\left[1-\left(\sin \left(\frac{n \pi}{2}\right)\right)^{2 n}+\left(\cos \left(\frac{n \pi}{2}\right)\right)^{2 n}\right]\right\}$

4. Using the virtual tool "Circle with Centre and Radius" construct the circles with centre at each above point and radius 0.3

5. Using "Object Properties" select the colour and the opacity of each circle (blue). This is achieved by clicking on the equation of each circle that are shown on Algebra window.

Demonstration: play with the slider by moving at the values 2, 3 and 4 and observe the dynamic of partitioning the set of 12 objects into 2, 3 and 4 groups, respectively. For each value of n, each group contains the same number of objects (Look at Fig.3).
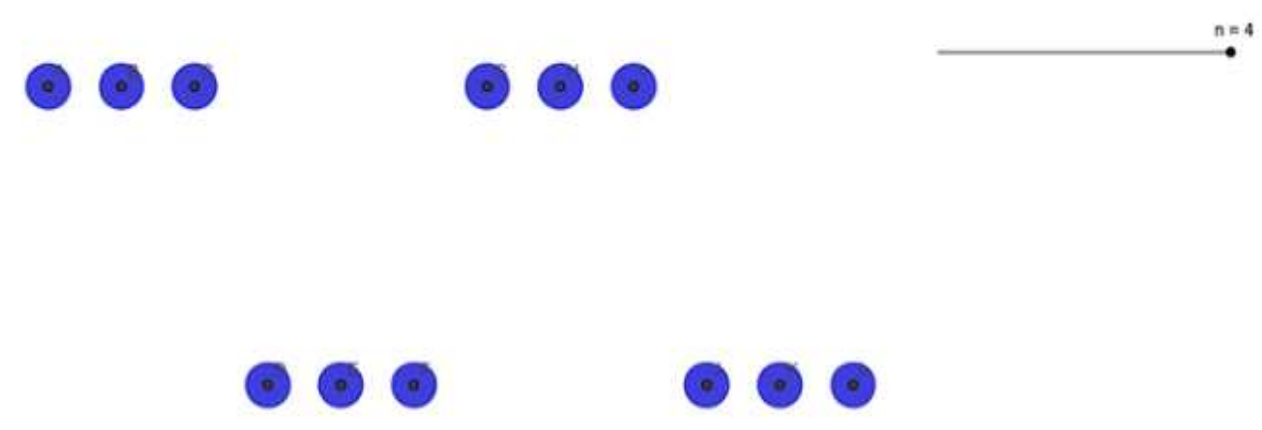

\section{$12: 4=3$}

Fig. 3. Twelve objects partitioned into 4 groups of equal size

\subsection{Representing the Equation of the Division in GeoGebra Window}

Show separately on GeoGebra window the following texts:

1. Using the virtual tool "ABC-Text" register the number 12 (representing the dividend) and the division operation, that is 12 :

2. Use again the "ABC-Text" tool to register variable $n$ as follows: open "Text" and, on the open window, click at Objects. On the displayed window of Objects choose the letter $\mathrm{n}$ (representing the divisor). If you register variable $\mathrm{n}$ in any other way (e.g. by typing $\mathrm{n}$ ) then it is not possible for $\mathrm{n}$ to take its values $1,2,3$ and 4 .

3. Using the virtual tool "ABC-Text" register the symbol of equality.

4. Enter in the Input field the function $\mathrm{j}=12 / \mathrm{n}$ or use the command Product $[12,1 / n]$ in order to display it on Algebra window.

5. Use again the "ABC-Text" tool to register letter $\mathrm{j}$ in the same way: open "Text" and on the open window click at Objects. On the displayed window of Objects choose the letter $\mathrm{j}$ (representing the quotient).

6. Arrange the texts in order they form the equation of the division by selecting the place, their size and their colour as desired.

When played with the slider and observed the dynamic of partitioning of the set of 12 objects into 2, 3 and 4 groups, respectively, simultaneously are shown the respective equations of the division.

Note 1: the respective applet is shared on GeoGebra tube.
You can download the free application from the GeoGebra Web site- www.geogebra.org and play with it by visiting http://www.geogebratube.org/material/show/id/134087 .

Note 2: since the division is the inverse of multiplication we can use the array table of multiplication to help students understand how division and multiplication are related. In multiplication is found the product of two factors, whereas in division is found the missing factor when the other factor and the product are known.

\subsection{The Measurement Division Modelling or Strategy}

Let see now the model related to the second concept of division, measurement. This is the case of determining the number of groups in which a given number of objects is equally shared or spread. In other words, the task is to find the number of groups (which is unknown) when the total number of the objects is shared in equal quantities (which is known).

The common strategy the students use is: they create sets containing the given number of objects (this number represents the equal quantity in each set) until they have used all the objects. Finally, they count how many sets they made. Recall that this is not an easy task for the students of elementary school. The strategy is based on using trial and error method to determine the right number of sets: the number of sets determined by the students can be less than the right one (there are objects left out), the number of sets determined by the students can be greater than the right one (there are sets with no objects) or, they can hit the right number of sets since the first trial. This type of strategy used 
by the students is called the measurement strategy, because the students in each trial measure (count) the number of sets they have created.

The measurement strategy relates to the common practice applied to find the number of containment of a given stick in a given straight part of a road, of a given segment in another bigger segment when the smaller segment is contained in it a whole number of times. The common practice is measuring the length of the straight part of the road, starting from one edge to the other, by the use of the stick as a unit. Assume our task is to find out how many times the segment (stick) OA is contained into the segment OP given that the length of segment OP is 14 units and the length of the stick is 2 units. The equivalent problem is: find out how many times number 2 is contained to number 14 , or what is $14: 2$ ? To solve the problem we measure the length of $\mathrm{OP}$, starting from point $\mathrm{O}$, and using the stick OA. The model and the respective strategy of answering the above question is shown in Fig.4.

The respective applet is shared on GeoGebra tube: http://tube.geogebra.org/material/show/id/165465

Read the instructions and download the applet to play with it.

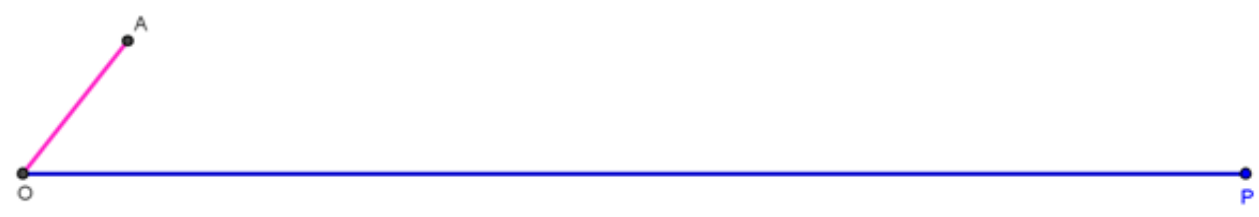

Fig. 4. The measurement model representing the division $14: 2$

\section{Conclusions}

Our research and practice drives our previous work. Our purpose is to conduct and utilize applied research in special areas of educational content, instruction and technology to improve student learning and enhance teaching and learning and meet the needs of diverse learners. Working alongside with the other researchers and GeoGebra specialists, the main objectives are:

- Help the teachers to use technology effectively in their teaching process to improve student learning

- Promote student learning by improving teaching practices and providing capacity-building solutions

- Increase teacher pedagogical content knowledge and improve instructional practice

- Encourage the teachers engage themselves in research activity and innovative educational practices and teaching strategies

- Encourage the teachers to use designed models, develop other models and share their works and results within GeoGebra community and with other education communities.

- Contribute in the professional development programs

Any teacher can learn and be equipped mostly on his/her own by experimentation, adding here the help from suggestions on GeoGebra wiki, Twitter, and googling stuff from the online help.

\section{Acknowledgement}

Lindita Kllogjeri thanks her husband, Pellumb Kllogjeri, for leading the Special Issue, titled "Growing GeoGebra Environment and Use", for the American Journal of Software Engineering and Applications, creating this way the opportunity that her contribution in teaching with technology be publicized.

\section{References}

[1] Carl Stitz, Ph.D. Jeff Zeager, Ph.D., "College Trigonometry", Lakeland Community College and Lorain County Community College, August 2010, pp. 600-700

[2] GeoGebra. Available via: http://www.geogebra.org; [Accessed 27 February 2009].

[3] HOHENWARTER JUDITH, HOHENWARTER MARKUS: "Introduction to GeoGebra", 2008, pp. 3 - 20.

[4] Hohenwarter M. \& Preiner J., "Dynamic mathematics with GeoGebra", TheJournal of Online Mathematics and its Applications, ID1448, vol. 7, 2007

[5] Hohenwarter, M., \&Lavicza, Z., "The strength of the community: How GeoGebra can inspire technology integration in mathematics teaching", Mathematics, Statistics, Operation Research Connections, 9(2), 2009, pp. 3-5.

[6] INFORMATION AND COMMUNICATION TECHNOLOGY IN EDUCATION (A CURRICULUM FOR SCHOOLS AND PROGRAMME OF TEACHER DEVELOPMENT), Division of Higher Education (C) UNESCO 2002, Printed in France, pp. $8-22$.

[7] Paul A. Foerster ,Trigonometry: Functions and Applications, published by Prentice Hall, USA 1990 